\title{
hVMAT2: A Target of Individualized Medication for Parkinson's Disease
}

\author{
Nian Xiong ${ }^{1}$ Nuomin $\mathrm{Li}^{2,3} \cdot$ Eden Martin ${ }^{4} \cdot$ Jinlong $\mathrm{Yu}^{3} \cdot \mathrm{Jie} \mathrm{Li}^{3,5} \cdot \mathrm{Jing} \mathrm{Liu}^{6}$ • \\ David Yue-Wei Lee ${ }^{6}$. Ole Isacson ${ }^{7}$ • Jeffery Vance $^{4}$ • Hong Qing ${ }^{2}$. Tao Wang ${ }^{1}$. \\ Zhicheng Lin $^{3}$
}

Published online: 2 May 2016

(C) The American Society for Experimental NeuroTherapeutics, Inc. 2016

\begin{abstract}
Vesicular monoamine transporter 2 (VMAT2) is responsible for sequestering cytosolically toxic dopamine into intracellular secretory vesicles. Animal genetic studies have suggested that reduced VMAT2 activity contributes to the genetic etiology of Parkinson's disease (PD), but this role has not been established in humans. Based on human genetic association and meta-analysis, we first confirm the human VMAT2 ( $h V M A T 2$ or $S L C 18 A 2)$ promoter as a risk factor for PD in both family and unrelated US white people: marker rs363324 at $-11.5 \mathrm{~kb}$ in the hVMAT2 promoter is reproducibly associated with PD in a cohort of nuclear families ( $p=0.04506$ in early-onset PD) and 3
\end{abstract}

Nian Xiong and Nuomin Li contributed equally to this work.

Electronic supplementary material The online version of this article (doi:10.1007/s13311-016-0435-5) contains supplementary material, which is available to authorized users.

Zhicheng Lin

zhicheng_lin@hms.harvard.edu

1 Department of Neurology, Union Hospital, Tongji Medical College, Huazhong University of Science and Technology, Wuhan 430074, Hubei, China

2 School of Life Sciences, Beijing Institute of Technology, 100081 Beijing, China

3 Laboratory of Psychiatric Neurogenomics, Division of Basic Neuroscience, McLean Hospital, Belmont, MA 02478, USA

4 Hussman Institute for Human Genomics, Miller School of Medicine, University of Miami, Miami, FL 33136, USA

5 Tianjin Mental Health Center, Tianjin Anding Hospital, 300222 Tianjin, China

6 Bio-Organic and Nutritional Products Laboratory, McLean Hospital, Belmont, MA 02478, USA

7 Neuroregeneration Laboratories, McLean Hospital, Belmont, MA 02478, USA unrelated US white people (meta-analysis $p=0.01879$ ). In SHSY5Y cells, low activity-associated $h V M A T 2$ promoter confers high methylpiperidinopyrazole iodide cytotoxicity, which is likely attributed to functional polymorphisms bound by nuclear proteins. Interestingly, treatments with the dopamine neuronprotecting agent puerarin upregulates the promoter activity in a haplotype- and cell line-dependent manner. These pharmacogenetic findings suggest that $h V M A T 2$ could be a risk factor and imply it as a target of genetic medications for PD.

Keywords Association · Epigenetics · Haplotypic upregulation $\cdot$ Haplotypic toxicity $\cdot$ Meta-analysis $\cdot$ Vesicular monoamine transporter $2 \cdot S L C 18 A 2$

\section{Introduction}

Parkinson's disease (PD) is the second most common neurodegenerative movement disorder, characterized clinically by resting tremor, akinesia, and rigidity. The main pathological finding associated with PD is degeneration of the dopaminergic neurons of the substantia nigra pars compacta (SNc), leading to loss of dopamine in the striatum and the presence of ubiquitin-/ $\alpha$-synuclein-positive cytoplasmic inclusions known as Lewy bodies [1-3]. The depletion of dopaminewhich is up to about $80-85 \%$ of the dopamine content in the striatum, while about $50-60 \%$ of the doparminergic neurons are lost in the substantia nigra $[4,5]$ — contributes to the motor symptoms of PD. However, cytosolic dopamine is metabolized by enzymatic deamination or broken down by autoxidation, producing reactive, harmful oxidative products [6], suggesting that packaging of the cytosolic dopamine molecules into vesicles be critical because it can prevent dopamine neurotoxicity in cytosol. 
Vesicular monoamine transporters (VMATs) do this critical packaging job and regulate vulnerability to PD. VMATs are part of the solute carrier family of transporters, taking up dopamine, as well as other monoamines (serotonin, epinephrine, and norepinephrine), into intracellular secretary vesicles. VMAT1 is expressed exclusively in the peripheral nervous system, while VMAT2 is expressed both in the peripheral and central nervous systems, and does the packaging job in monoaminergic systems in the brain. Previous studies have indicated that dysfunction of VMAT2 proteins can evoke cytoplasmic dopamine accumulation and finally leads to dopaminergic neuron death. Consistently, striatal VMAT2 protein expression and SNc VMAT2 mRNA level are significantly reduced in patients with $\mathrm{PD}[7,8]$. It has been suggested that the loss of VMAT2 function within the neuron is associated with the development of PD. In VMAT2 knockout mice, the VMAT2 protein density in heterozygous mice was decreased by half compared with wild-type mice and was undetectable in the homozygous mice. The homozygous mice died within a few days of birth, while the heterozygotes were viable to adulthood, without any histological abnormalities. However, the heterozygote knockout mice underwent progressive nigrostriatal degeneration, displayed motor symptoms of PD, and developed higher vulnerability to 1-methyl-4-phenyl-1,2, 3,6-tetrahydropyridine (MPTP) [9, 10]. Moreover, in VMAT2 gene-deficient mice, typical characteristics of $\mathrm{PD}$, including a large reduction in brain tissue monoamines, motor impairments, enhanced sensitivity to dopamine agonism, greater vulnerability to MPTP in terms of nigral dopamine cell death, $\alpha$ synuclein accumulation, and a levodopa-responsive behavioral deficit, could be observed $[11,12]$. Thus, these animal genetic studies suggest that genetic variation in VMAT2 activity may confer vulnerability for dopaminergic degeneration.

We, and others, have found natural products or alternative medicines, including puerarin, dl-3-n-butylphthalide (NBP), and edaravone, may protect dopaminergic neurons in various neurotoxin models of PD [13-17]. More interestingly, NBP and edaravone both upregulated VMAT2 gene activity, again suggesting that high VMAT2 activity protects dopaminergic neurons, and implying a therapeutic approach for PD. It remains to be understood whether puerarin's neuroprotective role is correlated with upregulation of VMAT2 gene activity. It is unknown either whether puerarin, like other drugs, can regulate $h V M A T 2$ in a variant-dependent manner [18].

Considering the neuroprotective effects of VMAT2 in preventing dopamine cytotoxicity and other pesticide toxicity to dopaminergic neurons, genomic variation affecting the function and expression of the human VMAT2 is likely associated with the susceptibility to PD [11]. Recently, genetic association studies have used genetic markers located in the promoter regions of $h V M A T 2$ and found that the human VMAT2 gene ( $h V M A T 2$ or SLC18A2) was associated with PD in US and Italian populations [18, 19]. These studies both showed positive associations and implied the promoter as a risk locus. Glatt et al. [20] first reported that core promoter haplotypes associated with high promoter activity were protective against PD as, statistically, they were over-represented in the controls comparing with the cases. It remains unknown whether lower-activity-associated haplotypes may actually confer high cytotoxicity.

Moreover, the Italian study found that the single nucleotide polymorphisms (SNPs) rs363371 and rs363324 were associated with PD in an unrelated cohort. Both SNPs were beyond the core promoter that Glatt et al. [20] studied. Overall, our resequencing data have suggested that there is greater diversity of haplotype-dependent transcriptional regulations, which affects the selection of $h V M A T 2$ in the human lineage [18]. Several promoter haplotypes have been selected, including A-G, with different regulatability, but it remains unclear whether these functionally different haplotypes, covering a $>$ $17.4 \mathrm{~kb}$ promoter, can contribute to cytotoxicity. A limitation in these 2 PD association studies is the modest sample sizes used (in the order of hundreds).

On the contrary, Kariya et al. [21] found that 2 polymorphic sequences upstream of the core $h V M A T 2$ promoter were not associated with PD in a Japanese population. Thus, these current $h V M A T 2$ genetic data add to our understanding of hVMAT2 structure and function by revealing polar diversity and functional significance of polymorphism in hVMAT2. However, the results of the studies are currently limited and warrant replications in more populations for reproducibility, comprehension, and functional clarification of the hVMAT2based genetic etiology of PD.

In this study, we confirmed the association of $h V M A T 2$ with idiopathic PD in both family and unrelated samples of different US white populations, and investigated whether different promoter haplotypes confer different levels of cytotoxicity and whether epigenetic activities correlated with hVMAT2 mRNA levels, and then exploited potentials of hVMAT2 as a medication target for PD.

\section{Methods}

\section{Family Study}

This study used 755 families with PD, who have been described previously [20]. In the 755 families, there were 1011 affected siblings, 1049 unaffected siblings, and 275 parents. In the 75 early-onset families, there were 100 affected siblings, 112 unaffected siblings, and 93 parents. The families were primarily discordant sibships with at least 1 affected and 2 unaffected sibling. Approximately $40 \%$ of the families were multiplex, with $>1$ affected sibling. All families were of European descent and known PD mutation carriers (Parkin, $S N C A$, and $L R R K 2$ ) were excluded from this study. 
Genotyping followed the same TaqMan allelic discrimination assay described in Oliveira et al. [22].

\section{Statistical Analysis of Family Genotype}

All genotypes were tested for deviations from HardyWeinberg equilibrium by using $\chi^{2}$ testing in both affected ( 1 affected from each family) and unaffected (1 unaffected from each family) groups by the Genetic Data Analysis program [23]. We performed the Association in the Presence of Linkage (APL) test for SNPs associated with PD risk [24]. We used the Monks test to test for association between the SNPs and age at onset (AAO) [24]. We considered individuals stratified by AAO (early- and late-onset) using two age cutoffs (40 and 60 years). Overall, the results from these tests provide us with a reliable evaluation of age-related association between SNPs and PD.

\section{Imputation-based Case-Control Analysis of Genotype and Phenotype Database Genome-wide Association Study Datasets}

To examine $h V M A T 2$ in sporadic PD samples, we consulted the Genotype and Phenotype Database for 3 independent case-control studies: phg000126.v1.p1 provided by the Center for Inherited Disease Research (CIDR); phs000089.v3.p2 provided by National Institute of Neurological Disorders and Stroke (NINDS); and phs000196.v2.p1 provided by the NeuroGenetics Research Consortium (NGRC). Based upon a published protocol [25], we removed discordant sex information, outlying or missing genotype or heterozygosity rates, duplicated or related individuals, and divergent ancestry. With these cleaned datasets, we performed phasing with Shapeit and imputation with Impute 2 by using the 1000 Genomes Project multipopulation panel macGT1 for missing genotypes in hVMAT2 [26, 27]. The PLINK package was used for data management, association, and meta-analyses (meta-analysis) [28].

\section{Different Promoter Activity and Cytotoxicity Conferred by Different Haplotypes in vitro}

As described previously, in phVMAT2-6.3A-luc, phVMAT26.3C-luc, and phVMAT2-6.3G-luc, hVMAT2 promoters drive the expression of the reporter firefly luciferase (Luc) for drug regulation studies [18].

VMAT2 expression plasmids were prepared as follows. The 1.57-kb hVMAT2 cDNA fragment tagged with HindIII and $F$ seI underwent 50 cycles of polymerase chain reaction (PCR) using Agilent's PfuUltra II Fusion HS DNA Polymerase and the associated protocol (Agilent Technologies, Santa Clara, CA, USA), an annealing temperature of $52^{\circ} \mathrm{C}$, and primers listed in Table 1. A cDNA template
Table 1 Oligonucleotides used in this study

\begin{tabular}{|c|c|}
\hline \multicolumn{2}{|l|}{ cDNA cloning } \\
\hline hvmat2-orf1 & aaaaaaagcttaagcgacccegagcggag \\
\hline hvmat2-orf2 & ttttggccggcetcagtcactttcagattctt \\
\hline \multicolumn{2}{|l|}{ EMSA } \\
\hline rs34074668-1f & agagagataattttatac \\
\hline rs34074668-1r & tttttgtataaaattatctctct \\
\hline rs34074668-2f & agagagataataattttatac \\
\hline rs34074668-2r & tttttgtataaaattattatctctct \\
\hline rs2619096tf & ggtatgattgatcctg \\
\hline rs2619096tr & tttttcaggatcaatcatacc \\
\hline rs2619096cf & ggtatgactgatcetg \\
\hline rs2619096cr & $\mathrm{tttttcaggatcagtcatacc}$ \\
\hline rs10689256-2f & tccctgtctgtgataactag \\
\hline rs10689256-2r & tttttctagttatcacagacaggga \\
\hline rs10689256-3f & tccctgtctgtgtgataactag \\
\hline rs10689256-3r & tttttctagttatcacacagacaggga \\
\hline \multicolumn{2}{|l|}{ qRT-PCR } \\
\hline hGAPDHf & tgecctcaacgaccactttg \\
\hline hGAPDHr & tctctcttcetcttgtgctcttgc \\
\hline hVMAT2f & cggatgtggcattttgtatgg \\
\hline hVMAT2r & ttcttctttggcaggtggacttc \\
\hline hSNCAf & tgtaggctccaaaaccaagg \\
\hline hSNCAr & ctgctccetccactgtcttc \\
\hline hDNMT1f1 & catgagtgcattggtggct \\
\hline hDNMT1r1 & gccatggtgccgtctga \\
\hline hDNMT3af2 & ttcgctaataaccacgacca \\
\hline hDNMT3ar2 & agcgattccatcaaagaga \\
\hline $\mathrm{hDAC} 3 \mathrm{f} 2$ & aaggaatttcttttcacctct \\
\hline $\mathrm{hDAC} 3 \mathrm{r} 2$ & gtctccagactctttccca \\
\hline hDAC6f1 & tggeccagaccatttctga \\
\hline hDAC6r1 & ggctcctcccacagtctcct \\
\hline hDAC11f2 & tggagctggeccttcct \\
\hline $\mathrm{hDAC} 11 \mathrm{r} 2$ & gggctggtgacttcgtgt \\
\hline hMYST2f2 & cttccacgaactcctcct \\
\hline hMYST2r2 & cttccttcacttgaacaaca \\
\hline hACTNBf & aactggaacggtgaaggtgacagc \\
\hline hACTNBr & ggtggcttttaggatggcaag \\
\hline rGAPDHf & atgactctacccacggcaag \\
\hline rGAPDHr & tactcagcaccagcatcacc \\
\hline $\mathrm{r} \beta$-actinf & gtcgtaccactggcattgtg \\
\hline $\mathrm{r} \beta$-actinr & tctcagctgtggtggtgaag \\
\hline rVMAT2f & agacgtggccttttgtatgg \\
\hline rVMAT2r & aagcagagtggagcaaaagc \\
\hline
\end{tabular}

EMSA = electrophoretic mobility shifting assay; qRT-PCR = real-time quantitative polymerase chain reaction

was prepared from RNA extracted from SH-SY5Y cells [18]. By the restriction sites HindIII and FseI, the cDNA fragment replaced the Luc gene $(l u c)$ in the 3 haplotypic promoter 
plasmids, resulting in 3 haplotypic hVMAT2 expression plasmids: phVMAT2-6.3A-hvmat2, phVMAT2-6.3C-hvmat2, and phVMAT2-6.3G-hvmat2.

Purified plasmid DNAs were introduced into SH-SY5Y cells using Superfect (Qiagen, Valencia, CA, USA), as described previously $[18,29]$. SH-SY5Y cells were cultured in Dulbecco's Modified Eagle's Medium containing 10\% fetal bovine serum (Life Technologies, Rochester, NY, USA) in 5\% $\mathrm{CO}_{2}$ and $100 \%$ humidity at $37^{\circ} \mathrm{C}$. Transfection in 24-well plates was prepared using $0.4 \mu \mathrm{g}$ plasmid DNA mixed briefly with $2.4 \mu 1$ Superfect (Qiagen). After 2 day's expression, transfected cells in each were washed with $1 \mathrm{ml}$ phosphatebuffered saline and harvested for Luc activity measurements using a Dual Luciferase Reporter System Kit (Promega, Madison, WI, USA), according to manufacturer's instructions.

For toxicity assay, cells transiently expressing $6.3-\mathrm{kb}$ haplotype-cDNA combinations were treated with $0.1 \mathrm{mM}$ methylpiperidinopyrazole iodide [MPP+ (Sigma-Aldrich, St. Louis, MO, USA) freshly prepared in $10 \mathrm{mM}$ Dulbecco's Modified Eagle's Medium as a stock solution] for 3 days, followed by cytotoxicity analysis using the CytoTox-ONE Homogeneous Membrane Integrity Assay (Promega). This assay fluorescently detected lactate dehydrogenase released from cells with damaged membrane, by using resazurin as the substrate. In the presence of lactate dehydrogenase, resazurin was converted into fluorescent resorufin in a quantitative manner. Triton X-lysed cells were used as "maximum" toxicity control $(100 \%)$, and relative fluorescence unit percentage of haplotype-expressing cells was expressed as cytotoxicity units.

\section{Electrophoretic Mobility Shifting Assay}

An electrophoretic mobility shifting assay was carried by using nuclear proteins extracted from SH-SY5Y cells, according to our previously published protocol $[18,30]$. Oligonucleotides used for annealing are listed in Table 1.

\section{Haplotypic Regulation by Drugs}

Puerarin was isolated from kudzu extract by countercurrent chromatography $[31,32]$. Its purity $(>99 \%)$ was verified by high-performance liquid chromatography analysis and structure verified by nuclear magnetic resonance spectroscopic analysis (Supplementary Fig. 1).

Transient expression of promoter haplotypes, treatments of the transient or stable expression with drugs, the isoflavone puerarin and the synthetic alkaloid levo-tetrahydropalmatine (1-THP) as a control, preparation of SH-SY5Y cell lines transiently or stably carrying $h V M A T 2$ promoter haplotypeLuc hybrids, and Luc assay procedures have been described in detail previously $[18,29]$. Briefly, 18-h treatments of expressing cells with $10 \mu \mathrm{M}$ agents, as indicated in Fig. 3, started $27 \mathrm{~h}$ after transfection (in case of transient expression). We measured Luc activity with a Luciferase Assay System Kit (Promega) in Bio-Tek Synergy HT/KC4 and a 96-well format. Cell density per well was estimated by protein amount based on Protein Assay Reagent (Bio-Rad, Hercules, CA, USA). An arbitrary unit of $h V M A T 2$ promoter activity was calculated as Luc activity: (readout/protein) (hVMAT2_MOCK) $_{\text {(readout/ }}$ protein) (Vector_MOCK), MOCK, transfection without DNA for a background control.

\section{In vitro and in vivo Upregulation of VMAT2 Expression by Puerarin}

Puerarin is a free radical scavenger that has been shown, via many studies $[13,15]$, to have neuroprotective effects. In this study, we explored the underlying mechanism of this neuroprotection by assessing the effect of puerarin on VMAT2 expression in vitro and in vivo.

\section{Quantitation of VMAT2 mRNA Levels by Real-Time Quantitative PCR}

After 4 human neuroblastoma cell lines (SH-SY5Y, IMR-32, SK-N-AS, and BE(2)-M17) were administered with puerarin $(100 \mu \mathrm{M})$ for $24 \mathrm{~h}$ we used TRIzol reagent (Life Technologies) to extract total RNA. Four rats from each group were injected intraperitoneally (i.p.) with vehicle (dimethyl sulfoxide with saline), or puerarin $(100 \mathrm{mg} / \mathrm{kg})$ twice, $18 \mathrm{~h}$ and $6 \mathrm{~h}$ before brain harvest. Live decapitation was used to euthanize all the rats, and left and right $\mathrm{SNc}$ regions ( -4.5 to $-6.2 \mathrm{~mm}$ caudal to the bregma) were micropunched. The tissues were added to $1.5-\mathrm{ml}$ RNasefree microfuge tubes with $100 \mu \mathrm{l}$ TRIzol reagent, then homogenized in ice by mechanical trituration, followed by the addition of $900 \mu \mathrm{l}$ TRIzol reagent to the tubes, to extract total RNA. Total RNA extraction from the cell lines, reverse transcription, and quantitative PCR have been described previously [18]. Briefly, reverse transcription was performed with Superscript II reverse transcriptase (Qiagen) oligo (dT) as the primer. Quantitative PCR was conducted with SYBR Green PCR Master Mix (Applied Biosystems, Foster City, CA, USA) and the primers listed in Table 1. Glyceraldehyde-3-phosphate dehydrogenase and $\beta$-actin were used as internal controls. VMAT2 primers showed amplification efficiency approximately equal (within 5\% difference) to that of the internal controls.

Immunohistochemical staining of VMAT2 protein levels in vivo. For the VMAT2 immunostaining, 4 more rats of each group were injected intraperitoneally with vehicle (dimethyl sulfoxide with saline) or puerarin $(100 \mathrm{mg} / \mathrm{kg})$ twice in a $24-\mathrm{h}$ period. Paraformaldehyde perfusion was used to sacrifice all the rats. Embedded with optimal cutting temperature compound, brain tissues were cut into serial 10 -mm-thick slices 
with a cryostat. Immunofluorescent staining was employed to label the VMAT2 (rabbit polyclonal antibody; SigmaAldrich) in the SNc (from $24.5 \mathrm{~mm}$ to $26.2 \mathrm{~mm}$ caudal to bregma). Expression was visualized by fluorescein isothiocyanate-conjugated goat-antimouse IgG (EMD Millipore, Billerica, MA, USA). To analyze VMAT2 expression densitometrically, a design-based unbiased stereological method and a morphometry/image analysis system (MacBiophotonics ImageJ, McMaster Biophotonics Facility, Hamilton, ON, Canada) were used to quantify protein density [33-35].

\section{Epigenetic Gene Expression in Human Dopaminergic Neurons}

Dopaminergic neurons were isolated randomly from the postmortem nigral tissue of 40 subjects ( 22 males, 18 females, mean age 58 years,), by laser capture microdissection [36]. Briefly, $10-\mu \mathrm{M}$ sections were immunostained with anti-tyrosine hydroxylase (TH) antibody and labeled neurons were captured via a PixCell II laser capture microdissection instrument (Arcturus, Mountain View, CA, USA). In total, 800-1000 random neurons were captured for each subject. RNA was extracted with an Arcturus PicoPure RNA Isolation Kit (ThermoFisher Waltham, MA, USA), followed by reverse transcription and real-time quantitative PCR for 6 human genes with primers listed in Table 1, as mentioned above.

\section{Results}

\section{hVMAT2 Association with PD in a Family Study}

Results from the family-based association tests for $5 \mathrm{hVMAT2}$ promoter markers typed in 755 families are, as listed in Table 2, either overall or stratified by the 2 AAO cut-offs. Of the 5 markers, only rs363371 at $-14.2 \mathrm{~kb}$ showed no association in either model, but the other 4 markers showed minimal positive associations in at least 2 conditions. Rs363324 at $-11.4 \mathrm{~kb}$ had a $p$-value of 0.045055 for eo40 by APL and 0.0283 for eo60 by Monks test. rs10689256 at -4.2 $\mathrm{kb}$ displayed an overall positive association $(p=0.0282$, Monks test); for AAO, this marker had a $p$-value of 0.016872 for eo40 by APL and of 0.0141 for eo60 by Monks test. Rs261906 at $-2.46 \mathrm{~kb}$ displayed an association pattern similar to rs10689256 and rs34074668 in a more proximal region, a pattern similar to rs363324 (Table 2). Overall, we saw nominal evidence of association with risk for 4/5 markers that are restricted to the early-onset strata (linkage disequilibrium could not be considered for association signals in a family-based study). For AAO, we saw an overall nominal association for $2 / 5$ markers, and significance increased only when late-onset individuals were excluded.

\section{Meta-analysis of $h V M A T 2$ Association with PD in Unrelated White American People}

Although the family data indicated association signals around the promoter regions, the marker density was only $0.4 / \mathrm{kb}$, which did not have high resolution. To replicate the finding independently, we consulted with the Genotype and Phenotype Database ( $\mathrm{dbGaP}$ ) datasets of 3 genome-wide association study (GWAS) cohorts, phg000126.v1.p1 (CIDR), phs000089.v3.p2 (NINDS), and phs000196.v2.p1 (NGRC), for hVMAT2 markers (approximately, from $20 \mathrm{~kb}$ upstream to $20 \mathrm{~kb}$ downstream). The CIDR dataset covered 344,301 SNPs in 900 cases and 867 controls; the NINDS dataset covered 572,140 SNPs in 940 cases and 801 controls; and the NGRC covered 1,012,895 SNPs in 2013 cases and 1995 controls. After applying genotype quality control as per the protocol of Anderson et al. [25], 79\% of the genotype information remained for the CIDR dataset, including 334,041 SNPs in 770 cases and 783 controls; $73 \%$ of the genotype information remained for the NINDS dataset, including 522,427 SNPs in 716 cases and 673 controls; and $73 \%$ of the genotype information remained for the NGRC PD environment dataset, including 817,332 SNPs in 2000 cases and 1986 controls. After genotype quality, imputation with a 1000-genome multipopulation panel and association analysis for each dataset (see Supplementary Fig. 2), association and meta-analyses were carried out.

As a result of the meta-analysis shown in Fig. 1 (detailed in Supplementary Table S1), the most robust signals were again revealed around the $-11 \mathrm{~kb}$ region. Interestingly, rs363324 was again among the associated markers in this region. The most significant marker was $\mathrm{rs} 10886050$ at $-8.026 \mathrm{~kb}(p=.007207$, odds ratio 0.89 ). The 3 proximal markers typed in the family sample were not picked in the imputation probably because a high threshold of 0.9 was used for high-quality imputation. However, there was also some evidence of association in the region around exon 2 . Together, the results from the association analyses in family and unrelated patients suggest that the promoter regions from rs2619103 at $-16.769 \mathrm{~kb}$ to rs 363332 at $2.083 \mathrm{~kb}$, including a distal region and core promoter regions, conferred modest but consistent genetic protection against PD. After stratification by sex, metaanalysis revealed that most of these association signals came from males rather than females, implying sex-dependence of $h V M A T 2$ related etiology (Supplementary Table S2). We have shown that the white promoter displayed high linkage disequilibrium, which would not help sort out underlying functional polymorphisms except for composing distinct haplotypes [37]. We thus took a haplotypic approach to functional analysis.

\section{Different Promoter Activities Conferred by Different Haplotypes in vitro}

Next, we asked whether proximal promoter haplotypes conferred varying activity and toxicity in the presence of the 
Table 2 hVMAT2 $(119,000,584-$ $119,038,941$ in chr10) promoter marker association with $\mathrm{PD}$ (risk $=\mathrm{APL}$ and $\mathrm{AAO}=$ Monk's) in US Caucasian nuclear families

\begin{tabular}{|c|c|c|c|c|c|}
\hline \multirow[t]{2}{*}{ SNP\# (alleles) BP } & \multirow[t]{2}{*}{ Stratification* } & \multirow[t]{2}{*}{ Nuclfams } & \multirow[t]{2}{*}{ Allele_freq } & \multicolumn{2}{|c|}{$P$-values from association tests } \\
\hline & & & & APL & Monks \\
\hline rs363371 & Overall & 734 & 0.229076 & 0.3214 & 0.8654 \\
\hline$(\mathrm{A} / \mathrm{G})$ & eo40 & 75 & 0.232207 & 0.11614 & 0.2982 \\
\hline 118986396 & 1040 & 659 & 0.228686 & 0.601914 & 0.3267 \\
\hline \multirow[t]{2}{*}[-14.188]{$\zeta$} & eo60 & 447 & 0.224322 & 0.326581 & 0.46 \\
\hline & lo60 & 287 & 0.236826 & 0.624936 & 0.4812 \\
\hline rs363324 & Overall & 730 & 0.294713 & 0.739958 & 0.0523 \\
\hline$(\mathrm{A} / \mathrm{G})$ & eo40 & 75 & 0.29139 & 0.045055 & 0.1446 \\
\hline 118989162 & 1040 & 655 & 0.295247 & 0.698848 & 0.6880 \\
\hline \multirow[t]{2}{*}[-11.422]{} & eo60 & 447 & 0.289271 & 0.31641 & 0.0283 \\
\hline & lo60 & 283 & 0.303743 & 0.524848 & 0.2628 \\
\hline rs10689256 & Overall & 731 & 0.303918 & 0.455899 & 0.0282 \\
\hline$(-/ \mathrm{TG})$ & eo40 & 76 & 0.311232 & 0.016872 & 0.0788 \\
\hline $118996351-2$ & 1040 & 655 & 0.302962 & 0.911098 & 0.6319 \\
\hline \multirow[t]{2}{*}[-4.233]{} & eo60 & 448 & 0.301251 & 0.203136 & 0.0141 \\
\hline & lo60 & 283 & 0.308828 & 0.827434 & 0.2913 \\
\hline rs 2619096 & Overall & 731 & 0.321018 & 0.501831 & 0.0476 \\
\hline$(\mathrm{C} / \mathrm{T})$ & eo40 & 75 & 0.333655 & 0.013646 & 0.1287 \\
\hline 118998127 & lo40 & 656 & 0.31938 & 0.833793 & 0.6598 \\
\hline \multirow[t]{2}{*}[-2.457]{} & eo60 & 447 & 0.316884 & 0.237695 & 0.0322 \\
\hline & lo60 & 284 & 0.328856 & 0.897904 & 0.1762 \\
\hline rs34074668 & Overall & 732 & 0.31724 & 0.828607 & 0.061 \\
\hline (-/TAA) & eo40 & 75 & 0.318634 & 0.031013 & 0.1088 \\
\hline $118998525-7$ & 1040 & 657 & 0.317143 & 0.602034 & 0.6086 \\
\hline \multirow[t]{2}{*}[-2.059]{} & eo60 & 449 & 0.313661 & 0.357332 & 0.0479 \\
\hline & lo60 & 283 & 0.324404 & 0.579364 & 0.3383 \\
\hline
\end{tabular}

\# rs\#/alleles/BP by GRCh37.p13;

*Age stratification: cut-off 40 years: eo40 (AAO<40), lo40 (AAO $\geq 40)$; cut-off 60 years: eo60 (AAO $<60)$, lo60 $(\mathrm{AAO} \geq 60)$

$\zeta$ Position in promoter (transcription start site $=+0.001 \mathrm{~kb}$ )

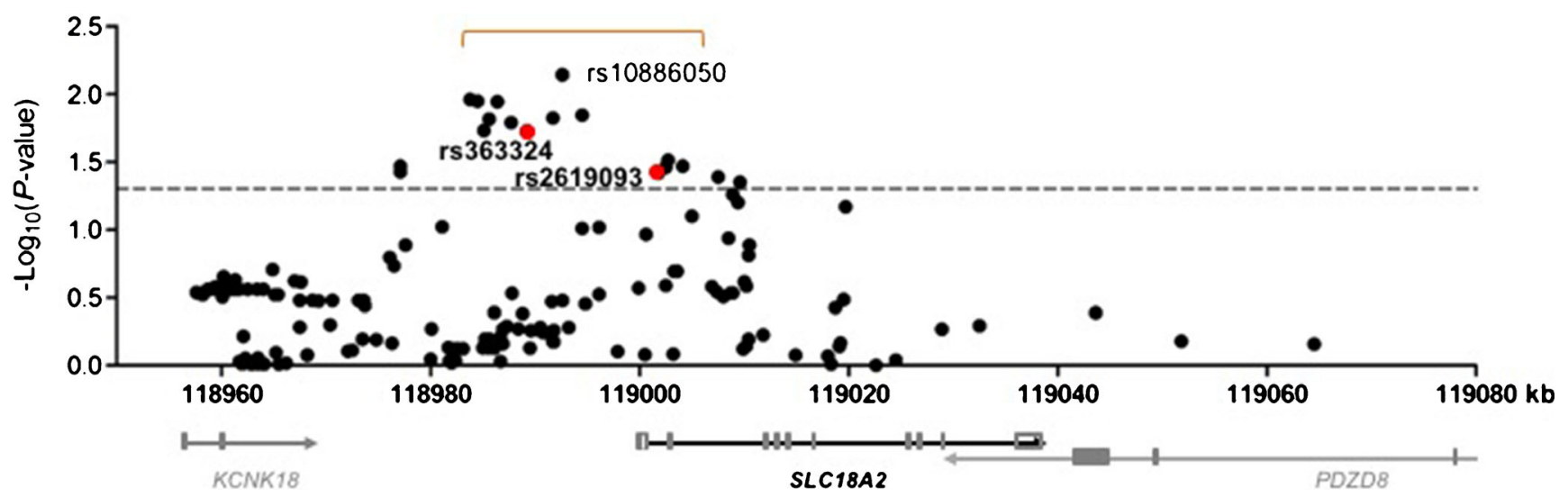

Fig. 1 Meta-analysis of human vesicular monoamine transporter-2 gene ( $h$ VMAT2/SLC18A2) association with Parkinson's disease in unrelated white Americans. Red dots indicate positive associations replicated by the family study; brackets indicate positive promoter association regions; dashed horizontal line indicate a $p$-value of 0.05 . Locations of $h V M A T 2$ in upstream and downstream genes in chromosome 10 (with coordinate) are indicated at the bottom 
neurotoxin MPP+. We used the Luc reporter and human neuroblastoma line SH-SY5Y as expression systems in which to examine the promoter activity of 3 different $6.3-\mathrm{kb}$ promoter haplotypes, A, C, and G (Fig. 2A), and to interrogate their ability to confer dopaminergic vulnerability. These 3 haplotypes contained the last 3 associated markers (from $-4.3 \mathrm{~kb}$ to $-2.1 \mathrm{~kb}$ ) identified by the family study (Table 2 ). As a result, Luc activity indicated C $>\mathrm{A}>\mathrm{G}$ in promoter activity (Fig. 2B). Consistently, after luc was replaced with hVMAT2 cDNA, mRNA levels and $\left[{ }^{3} \mathrm{H}\right]$ dopamine update activities were also $\mathrm{C}>\mathrm{A}>\mathrm{G}$ (Fig. $2 \mathrm{C}$, D). Inversely, the conferred vulnerability in the presence of the neurotoxin MPP+ was $\mathrm{C}<\mathrm{A}<\mathrm{G}$ (Fig. 2E). The 3 PD-related markers, rs 10689256, rs2619096, and rs34074668, might have contributed to haplotype-dependent risks because nuclear proteins differentially bound to the 2 alleles of each of the 3 markers (Fig. 2F). These in vitro functional assays suggested that functionally different promoter haplotypes conferred different levels of vulnerability in dopaminergic neurons, which is consistent

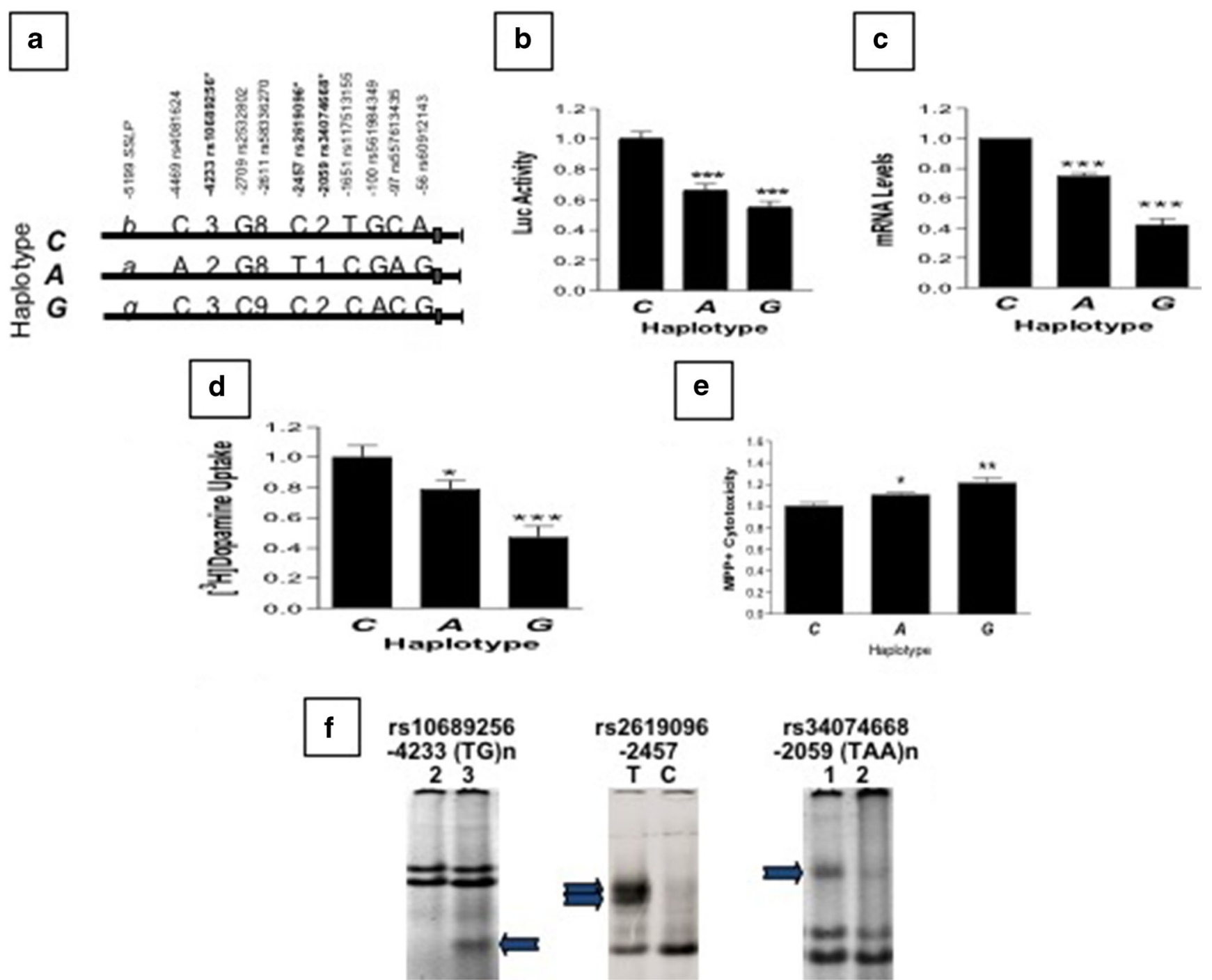

Fig. 2 Negative correlation between promoter activities and methylpiperidinopyrazole iodide (MPP + ) cytotoxicity conferred by 3 haplotypes $(\mathrm{C}, \mathrm{A}, \mathrm{G})$ of the $6.3-\mathrm{kb}$ hVMAT2 promoter region in $\mathrm{SH}-$ SY5Y. (A) Allelic differences among 3 promoter haplotypes, with markers indicated on top. Asterisk indicates 3 haplotypes associated with Parkinson's disease by family study (Table 2). (B) Three haplotypes showed various promoter activities, based on luciferase (Luc) reporter $\left(*^{* *} p<0.001\right.$ by Student's $t$ tests, compared with haplotype C. Three haplotypes drove hVMAT2 mRNA expression (C) or $\left[{ }^{3} \mathrm{H}\right]$ dopamine uptake activities (D) at levels consistent with Luc activities in (B), based on quantitative real-time polymerase chain reaction). ${ }^{\# \# \#} p<0.001$ compared with $\mathrm{C}$, based on ANOVA analysis. (E) Low activityassociated $h V M A T 2$ promoter confers high MPP+ cytotoxicity. ${ }^{*} p<0.05 ;{ }^{* *} p<0.01$ by $t$ tests, compared with C. Cells transiently expressing 6.3kb-cDNA hybrids were treated with $0.1 \mathrm{mM} \mathrm{MPP}+$ for 3 days, followed by cytotoxicity analysis using CytoTox-ONE Homogeneous Membrane Integrity Assay (Promega, Madison, WI, USA). (F) Three associated hVMAT2 promoter polymorphisms showed allele-dependent binding activity (indicated by arrows) by nuclear proteins isolated from SH-SY5Y, based on electrophoretic mobility shifting assay. Two non-single nucleotide polymorphisms are at -4233 base pairs (bp) $(n=2 / 3)$ and at $-2059 \mathrm{bp}(\mathrm{n}=1 / 2)$ 
with a clinical report that a defect in VMAT2 may be an early abnormality promoting mechanisms leading to nigrostriatal dopaminergic neuron death in $\mathrm{PD}$ [38].

\section{Epigenetic Correlation with $h V M A T 2$ mRNA Levels in Isolated Human Dopaminergic Neurons}

Extensive GWAS failed to pick up $h V M A T 2$ as a risk factor for PD [39], which is inconsistent with a pharmacogenetic view that VMAT2 plays an important role in protecting the dopaminergic neurons [40]. This contradiction suggests the possibility that epigenetic activity plays a more important role in regulating expression than DNA sequence variation. To explore this possibility, we investigated, for the first time, whether any related gene expression levels were correlated with hVAMT2 mRNA levels in human postmortem dopaminergic neurons. We studied 6 genes: $h D N M T 1, h D N M T 3 a, H D A C 3$, HDAC11, HDAC6, and hMYST2. These 6 were selected based on their relatively high expression levels in mouse dopaminergic neurons among known epigenetic genes [41]. Overall, the examined mRNA levels of all 7 genes, including $h V M A T 2$, were very low for $2 / 3$ of the patients. Supplementary Figure S3 shows a 1-phase exponential decay correlation between $h V M A T 2$ and $h D N M T 1$ mRNA levels. In addition, this negative correlation was also observed for $h D N M T 3 a$, and HDAC6 in either 20 controls or all 40 patients, but no correlation was observed for the 3 other genes related to histone acetylation (data not shown). No positive correlation was observed for any of these 6 epigenetic genes. These correlations were not significant but did suggest that the expression level of WVMAT2 could be regulated by epigenetic modifications besides DNA sequence variation. Specifically, the epigenetic activity of $h V M A T 2$ could be regulated more by DNA methylation than by histone acetylation in human dopaminergic neurons. DNA methylation profiling of $h V M A T 2$ in isolated dopaminergic neurons is required, but these epigenetic correlations may indicate an independent contribution of $h V M A T 2$ to PD etiology.

\section{Haplotype-dependent Upregulation of Promoter Activity by Dopaminergic Puerarin in vitro is Neuroprotective}

Given the haplotype-dependent vulnerability data, we examined whether the risk of low activity-associated haplotypes A and $G$ could be upregulated in promoter activity by potential therapeutics. In previous studies, we and others have shown that puerarin, a natural product extracted from Pueraria lobata used in Chinese traditional medicine, was able to protect dopaminergic neurons against neurotoxins in various PD models. We tried to address this question in both transiently (Fig. 3A, B) and stably (Fig. 3C) expressing SH-SY5Y cells. Stable expression was used for more reproducibility of regulation results. Puerarin treatment $(10 \mu \mathrm{M}$ for $18 \mathrm{~h})$ upregulated haplotype A 1.44-fold but not haplotype $\mathrm{G}$ in a transient expression system. In stably expressing cells, puerarin, but not the structurally related analog 1-THP, upregulated haplotype A 1.73-fold.

It remained to be seen whether the dopaminergic neuron protector puerarin was able to upregulate $h V M A T 2$ in chromosome 10 endogenously or rodent VMAT2 in vivo. In 4 human neuroblastoma cell lines, puerarin upregulated hVMAT2 mRNA levels in 2 of the cell lines, SH-SY5Y (1.39-fold) and SK-N-AS (1.42-fold), but not in IMR-32 or BE(2)-M17 (Fig. 3D). Puerarin regulated synuclein- $\alpha$ mRNA in none of these cell lines. Taken together, these in vitro functional data suggest that puerarin treatment of risk $h V M A T 2$ promoter variants could be haplotype dependent.

\section{Upregulation of VMAT2 Expression by Puerarin in vivo is also Neuroprotective}

Our previous study indicated that puerarin can protect dopaminergic neurons against rotenone toxicity and decrease the abnormal protein overexpressing in PD animal models. To further explore the underlying mechanism, in rat midbrain $\mathrm{SNc}$ tissue, puerarin treatment $(100 \mathrm{mg} / \mathrm{kg}$, i.p.) upregulated VMAT2 mRNA levels by 1.46- or 1.45 -fold (normalized by glyceraldehyde-3-phosphate dehydrogenase or $\beta$-actin; Fig. 4A). Moreover, immunostaining of VMAT2 showed that VMAT2 protein expression in the dopaminergic neurons of the puerarin-treated rats was upregulated by 1.72 -fold compared with the vehicle-treated rats (Fig. 4B-H). Together, puerarin upregulated both VMAT2 mRNA and protein levels in vivo.

\section{Discussion}

In this study, we found that 2 promoter regions of $h V M A T 2,1$ distal $(-11.422 \mathrm{~kb})$ and another more proximal (from -4.223 $\mathrm{kb}$ to $-2.059 \mathrm{~kb}$ ), are modestly and consistently associated with PD, based on both family and unrelated samples. For the first time, we show that low-activity haplotypes may confer more vulnerability in the presence of a neurotoxin and that hVMAT2 activity could be inhibited by DNA methylation in human dopaminergic neurons. Furthermore, upregulation of hVMAT2 promoter activity by potential therapeutics can be haplotype-dependent.

Although several promoter markers show association signals, none survived multiple-testing corrections, even for the relatively small numbers of SNPs examined herein, which is consistent with the fact that $h V M A T 2$ has never been picked up by any GWAS. Rs363324 at $-11.422 \mathrm{~kb}$ was implicated by both family and unrelated samples, although the imputation failed to include the 3 proximal promoter markers typed and implicated by the family study. Rs363371 at $-14.188 \mathrm{~kb}$, 


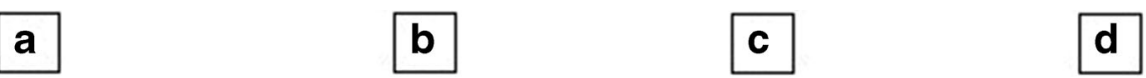

transient expression

stable expression

endogenous expression
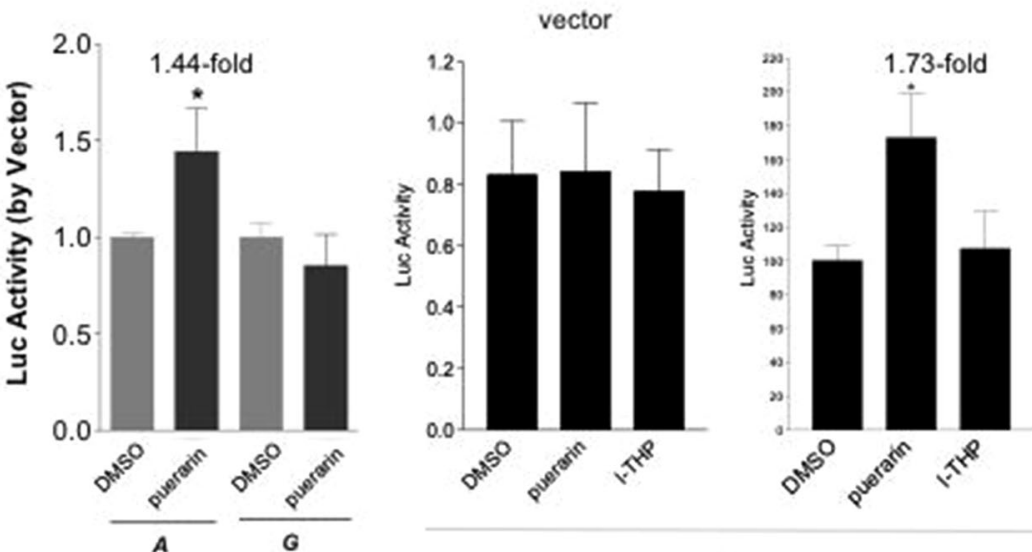

A

hVMAT2 promoter haplotype

Fig. 3 Individualized upregulation of $h V M A T 2$ by puerarin. (A) Haplotype-dependent regulation of $6.3-\mathrm{kb}$ promoter haplotype $A$ by puerarin based on transient expression in SH-SY5Y where dimethyl sulfoxide (DMSO) was the solvent (ANOVA $p=0.0242$; Student's $t$ tests $p=0.0385, n=6$ ). (B) Drug regulation of luciferase (Luc) activity transiently expressed from the vector pGL4.14, controlling for $h V M A T 2$ specificity in SH-SY5Y $(n=4)$. All drug treatments were $10 \mu \mathrm{M}$ for $18 \mathrm{~h}$.

which was not noticed in the family study, was associated with PD in the meta-analysis. A recent Italian study of unrelated patients has also indicated that both rs363324 and rs363371 are reproducibly associated with PD [19]. Both rs363371 and rs363324 have also been associated with alcoholism [37]. Together, the distal rs363324 is most consistently associated with PD (3 independent studies consisting of 5 independent cohorts).

It is still not clear whether the core promoter regions are consistently associated with PD. Our association studies did not show this, but the functional analysis supported the notion that low activity-associated haplotypes represent risk variants in this gene. This has been previously reported for a core promoter [20]. Together, these association and functional results were consistent with the involvement of the core promoter in the genetic etiology of $h V M A T 2$ in PD. In addition, for the first time, imputation analysis helps reveal a minor association region around exon 1 . However, these markers around the core regions should be typed in family samples, as well as for independent replications.

These modest association signals are appreciated because they support the extensive findings from classical gene knockout mouse and pharmacology studies. The heterozygote mice carrying a deletion of 1 copy of the gene displayed increased sensitivity to PD-related neurotoxin, MPTP [9, 42, 43]. Mice with significantly reduced VMAT2 activity also showed some PD symptoms, further supporting the pharmacological role of
VMAT2 in PD. Therefore, findings from different approaches, pharmacology, animal genetics, human genetics, and pharmacogenetics all indicate the protective role of $h V M A T 2$ in PD.

We have carried out the first converging study of hVMAT2 in PD and implicated the proximal promoter association by all 3 common markers typed, which is consistent with reported core promoter associations in unrelated patients [20]. These association findings suggest that $h V M A T 2$ contributes more to early-onset (especially $<40$ years old) PD than to cases with onset after the age of 60 years. Early-onset cases are usually attributable to familial mutations in known genetic risks [44, 45]. Conceivably, dopamine is an endogenous toxin for dopaminergic neurons, and high cytosolic dopamine concentrations kept for tens of years may contribute to dopaminergic neuron degeneration due to genetic "defect" in VMAT2 activity $[6,46]$. There might be a VMAT2-related early-onset pathway that connects known genetic risks such as synuclein- $\alpha$ and Parkin $[47,48]$. In future studies, it will be interesting to examine whether increased VMAT2 activity can attenuate the risks of known PD genes of early onset.

Moreover, the Italian and USA studies have both shown that $h V M A T 2$ is associated with PD in white populations by using genetic markers located in the promoter regions of hVMAT2 $[19,20]$. On the contrary, the Japanese study failed to find any association between 2 polymorphic sequences upstream of the core promoter and PD in the Asian population [21]. Besides ethnicity, a different sex distribution might have 
Fig. 4 Puerarin increased the vesicular monoamine transporter2 (VMAT2) mRNA (A) $\left[^{*} p<\right.$

0.05 compared with those in vehicle group tissue, normalized by $\beta$-actin or Glyceraldehyde-3phosphate dehydrogenase (GAPDH)] and protein levels (B$\mathrm{H})$ in rat midbrain substantia nigra pars compacta $(\mathrm{SNc})$. (B) Quantification of VMAT2 immunostaining in different groups, which showed that VMAT2 was upregulated 1.72fold in the SNc of puerarin rats, compared with the vehicle rats. $\left({ }^{*} p<0.05\right.$ compared with the VMAT2 protein level in the respective control SNc). (C-H) VMAT2 immunostaining in the $\mathrm{SNc}$ of vehicle and puerarin rats, respectively. The nuclear was stained by 4',6-diamidino-2phenylindole (DAPI; scale bar $=$ $20 \mu \mathrm{m}) \mathrm{SNCA}=\alpha$-synuclein

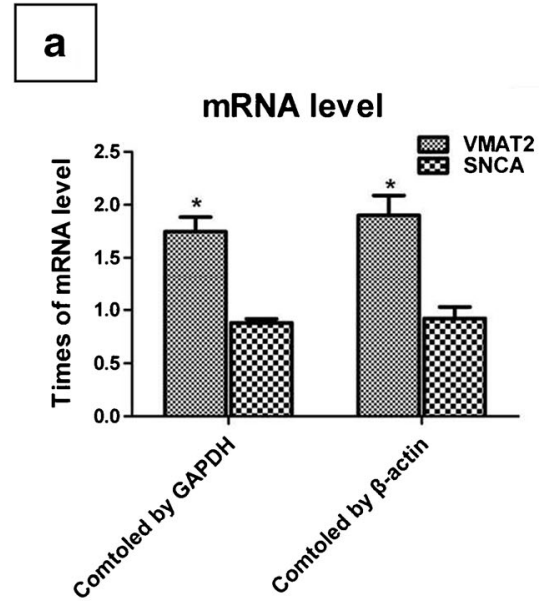

\section{b}
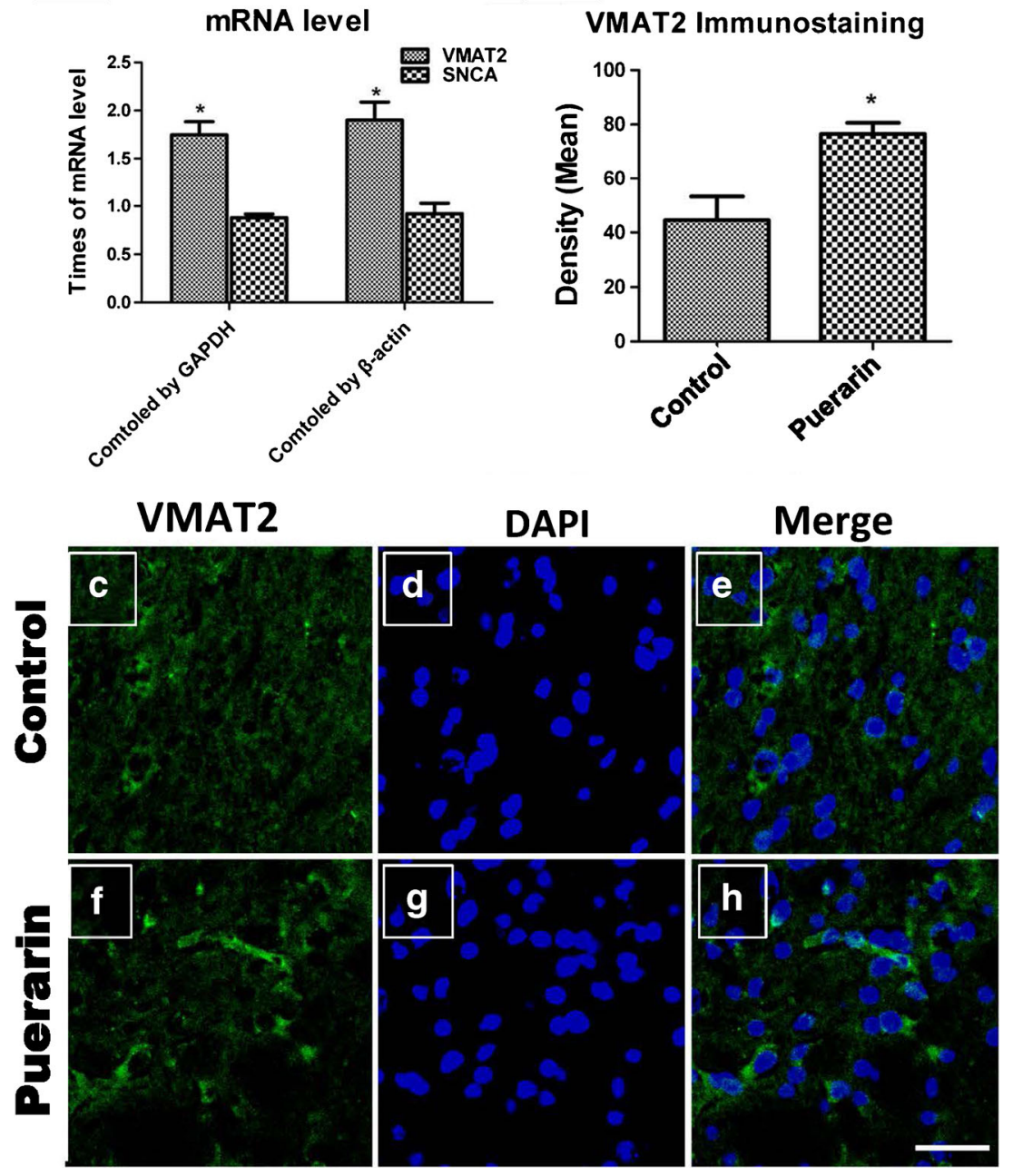

accounted for this difference, because the white populations studied were slightly male predominant, while the Japanese population was slightly female predominant, implying a possible sex $\times$ gender interaction. To further assess the sexdependence of $h V M A T 2$-related etiology, the meta-analysis showed consistently that most of these association signals came from males rather than females (Supplementary Table S2), supporting the observed male predominance of PD prevalence [49]. However, in these 2 white PD association studies, the sample sizes used were modest (in the order of hundreds). Thus, the sex-dependence of $h V M A T 2$-related etiology needs large cohorts for further validation, and for considering ethnicity, age, and environment.

In various PD models, we have shown that alternative medicines that can protect dopaminergic neurons are able to upregulate VMAT2 activity, in vivo and in vitro $[15,16,50]$. In this study, neuroprotective puerarin can upregulate VMAT2 in vivo. Most interestingly, upregulation was cell line- or haplotype-dependent (Fig. 3). It thus reaches a consensus that patients with sporadic PD may carry low activity-associated hVMAT2 promoter haplotypes. A pharmacogenetic approach to this genetic etiology is to upregulate hVMAT2 activity. However, this upregulation can depend upon the haplotypes carried in the patients. Based on the effect size and wellestablished role of VMAT2 in PD [40], we would like to suggest the use of $h V M A T 2$ upregulators as an adjunct therapeutic in individualized treatment of PD [51].

Currently there is no information on the mechanisms of action of puerarin. We carried out pathway analysis of puerarin but found no pathway between puerarin and hVMAT2, warranting future dissection of puerarin targets. We, and others, have shown $h V M A T 2$ associations with both PD and alcoholism [20, 37, 40, 52]. Interestingly, puerarin also displays therapeutic effects on these 2 dopamine-related diseases $[13,16,53,54]$. These dual-therapeutic effects are consistent with reports that alcoholism might have comorbidity with PD [55-57]. As there is no evidence for alcohol toxic contribution to dopaminergic neuron degeneration, reduced 
hVMAT2 expression likely confers vulnerability to alcohol abuse and PD. In this regard, puerarin may be promising for development into a dual therapeutic.

Although multiple approaches were used and consistent results obtained, limited numbers of markers were typed in the family and GWAS. Because of this, imputation of the GWAS datasets was unable to capture all markers used in the family study. Future studies need to type the metaanalysis-revealed positive markers, including rs10886050 in the family sample and vice versa. It remains unknown whether rs10886050 is functional or which proteins bind to the associated promoter polymorphisms (Fig. 2F). Environmental factors were not considered in the association analyses, which might have contributed to the genetic insignificance as PD is an environmentally related disease. Finally, the epigenetic findings of the $h V M A T 2$ activity need replication in independent cohorts.

In summary, VMAT2 is one of a few proteins converging supporting evidence from multiple approaches in the etiology of PD. The $h V M A T 2$ promoter is reproducibly associated with idiopathic PD without considering the potential epigenetic effects, such as DNA methylation. Low-activity promoter haplotypes may confer vulnerability for dopaminergic neurons so that this gene may be one of the biomarkers. Most importantly, haplotype-dependent upregulators of $h V M A T 2$ activity are promising genetic therapeutics for PD.

Acknowledgments We are grateful to $\mathrm{dbGaP}$ for granting $\mathrm{ZL}$ access to the GWAS datasets (Project\# 1542), and to the Harvard Brain Tissue Resource Center [National Institutes for Health $(\mathrm{NIH})$ ] for providing the human brain tissue samples for these investigations. This work was supported, in part, by research funding from Michael J. Fox Research Foundation, American Parkinson's Disease Association, and NIDA DA031573 and DA021409 grants (ZL),; NIH Morris K. Udall Parkinson's Disease Research Centers of Excellence P50NS39793 (OI) and 2P50NS071674 (JMV, ERM); Consolidated Anti-Aging Foundation, the Orchard Foundation, the Cooper Family and the Hansen Family (OI); and from the National Natural Science Foundation of China 81200983 (NX).

\section{References}

1. Gibb WR, Lees AJ. The relevance of the Lewy body to the pathogenesis of idiopathic Parkinson's disease. J Neurol Neurosurg Psychiatry 1988;51:745-752.

2. Leroy E, Boyer R, Auburger G, et al. The ubiquitin pathway in Parkinson's disease. Nature 1998;395:451-452.

3. Chung KK, Zhang Y, Lim KL, et al. Parkin ubiquitinates the alphasynuclein-interacting protein, synphilin-1: implications for Lewybody formation in Parkinson disease. Nat Med 2001;7:1144-1150.

4. Lang AE, Lozano AM. Parkinson's disease. First of two parts. N Engl J Med 1998;339:1044-1053.

5. Ross GW, Petrovitch H, Abbott RD, et al. Parkinsonian signs and substantia nigra neuron density in decendents elders without PD. Ann Neurol 2004;56:532-539.
6. Chen L, Ding Y, Cagniard B, et al. Unregulated cytosolic dopamine causes neurodegeneration associated with oxidative stress in mice. $\mathrm{J}$ Neurosci 2008;28:425-433.

7. Okamura N, Villemagne VL, Drago J, et al. In vivo measurement of vesicular monoamine transporter type 2 density in Parkinson disease with (18)F-AV-133. J Nuclear Med 2010;51:223-228.

8. Harrington KA, Augood SJ, Kingsbury AE, Foster OJ, Emson PC. Dopamine transporter (Dat) and synaptic vesicle amine transporter (VMAT2) gene expression in the substantia nigra of control and Parkinson's disease. Brain Res Mol Brain Res 1996;36:157-162.

9. Takahashi N, Miner LL, Sora I, et al. VMAT2 knockout mice: heterozygotes display reduced amphetamine-conditioned reward, enhanced amphetamine locomotion, and enhanced MPTP toxicity. Proc Natl Acad Sci U S A 1997;94:9938-9943.

10. Wang YM, Gainetdinov RR, Fumagalli F, et al. Knockout of the vesicular monoamine transporter 2 gene results in neonatal death and supersensitivity to cocaine and amphetamine. Neuron 1997;19: 1285-1296.

11. Caudle WM, Richardson JR, Wang MZ, et al. Reduced vesicular storage of dopamine causes progressive nigrostriatal neurodegeneration. J Neurosci 2007;27:8138-8148.

12. Mooslehner KA, Chan PM, Xu W, et al. Mice with very low expression of the vesicular monoamine transporter 2 gene survive into adulthood: potential mouse model for parkinsonism. Mol Cell Biol 2001;21:5321-5331.

13. Li X, Sun S, Tong E. Experimental study on the protective effect of puerarin to Parkinson disease. J Huazhong Univ Sci Technolog Med Sci 2003;23:148-150.

14. Cheng YF, Zhu GQ, Wang M, et al. Involvement of ubiquitin proteasome system in protective mechanisms of Puerarin to $\mathrm{MPP}(+)-$ elicited apoptosis. Neurosci Res 2009;63:52-58.

15. Zhang X, Xiong J, Liu S, et al. Puerarin protects dopaminergic neurons in Parkinson's disease models. Neuroscience 2014;280: 88-98.

16. Xiong N, Huang J, Chen C, et al. Dl-3-n-butylphthalide, a natural antioxidant, protects dopamine neurons in rotenone models for Parkinson's disease. Neurobiol Aging 2012;33:1777-1791.

17. Xiong N, Xiong J, Khare G, et al. Edaravone guards dopamine neurons in a rotenone model for Parkinson's disease. PLOS ONE 2011;6:e20677.

18. Lin Z, Zhao Y, Chung CY, et al. High regulatability favors genetic selection in SLC18A2, a vesicular monoamine transporter essential for life. FASEB J 2010;24:2191-2200.

19. Brighina L, Riva C, Bertola F, et al. Analysis of vesicular monoamine transporter 2 polymorphisms in Parkinson's disease. Neurobiol Aging 2013;34:1712 e9-13.

20. Glatt CE, Wahner AD, White DJ, Ruiz-Linares A, Ritz B. Gain-offunction haplotypes in the vesicular monoamine transporter promoter are protective for Parkinson disease in women. Hum Mol Genet 2006;15:299-305.

21. Kariya S, Hirano M, Takahashi N, Furiya Y, Ueno S. Lack of association between polymorphic microsatellites of the VMAT2 gene and Parkinson's disease in Japan. J Neurol Sci 2005;232:9194.

22. Oliveira SA, Li YJ, Noureddine MA, et al. Identification of risk and age-at-onset genes on chromosome 1p in Parkinson disease. Am J Hum Genet 2005;77:252-264.

23. Zaykin D, Zhivotovsky L, Weir BS. Exact tests for association between alleles at arbitrary numbers of loci. Genetica 1995;96(12):169-178.

24. Monks SA, Kaplan NL. Removing the sampling restrictions from family-based tests of association for a quantitative-trait locus. Am J Hum Genet 2000;66:576-592.

25. Anderson CA, Pettersson FH, Clarke GM, Cardon LR, Morris AP, Zondervan KT. Data quality control in genetic case-control association studies. Nat Protoc 2010;5:1564-1573. 
26. Delaneau O, Marchini J, Zagury JF. A linear complexity phasing method for thousands of genomes. Nat Methods 2012;9:179-181.

27. Marchini J, Howie B, Myers S, McVean G, Donnelly P. A new multipoint method for genome-wide association studies by imputation of genotypes. Nat Genet 2007;39:906-913.

28. Purcell S, Neale B, Todd-Brown K, et al. PLINK: a tool set for whole-genome association and population-based linkage analyses. Am J Hum Genet 2007;81:559-575.

29. Zhao Y, Xiong N, Liu Y, et al. Human dopamine transporter gene: differential regulation of 18-kb haplotypes. Pharmacogenomics 2013;14:1481-1494.

30. Zhao Y, Zhou Y, Xiong N, Lin Z. Identification of an intronic cisacting element in the human dopamine transporter gene. Mol Biol Rep 2012;39:5393-5399.

31. Lee YW CC, Fang QC, Ito Y The application of true countercurrent chromatography in the isolation of natural products. J Nat Prod 1989;52:5.

32. Ma Z, Wu Q, Lee DY, Tracy M, Lukas SE. Determination of puerarin in human plasma by high performance liquid chromatography. J Chromatogr B 2005;823:108-114.

33. Sarugaser R, Lickorish D, Baksh D, Hosseini MM, Davies JE. Human umbilical cord perivascular (HUCPV) cells: a source of mesenchymal progenitors. Stem Cells 2005;23:220-229.

34. Xiong N, Cao X, Zhang Z, et al. Long-term efficacy and safety of human umbilical cord mesenchymal stromal cells in rotenoneinduced hemiparkinsonian rats. Biol Blood Marrow Transplant 2010;16:1519-1529.

35. Xiong N, Zhang Z, Huang J, et al. VEGF-expressing human umbilical cord mesenchymal stem cells, an improved therapy strategy for Parkinson's disease. Gene Ther 2011;18:394-402.

36. Kennedy JL, Xiong N, Yu J, et al. Increased nigral SLC6A3 activity in schizophrenia patients: findings from the Toronto-McLean cohorts. Schizophr Bull 2015 Dec 26 [Epub ahead of print].

37. Lin Z, Walther D, Yu XY, Li S, Drgon T, Uhl GR. SLC18A2 promoter haplotypes and identification of a novel protective factor against alcoholism. Hum Mol Genet 2005;14:1393-1404.

38. Pifl C, Rajput A, Reither H, et al. Is Parkinson's disease a vesicular dopamine storage disorder? Evidence from a study in isolated synaptic vesicles of human and nonhuman primate striatum. J Neurosci 2014;34:8210-8218

39. Nalls MA, Pankratz N, Lill CM, et al. Large-scale meta-analysis of genome-wide association data identifies six new risk loci for Parkinson's disease. Nat Genet 2014;46:989-993.

40. Lohr KM, Bernstein AI, Stout KA, et al. Increased vesicular monoamine transporter enhances dopamine release and opposes Parkinson disease-related neurodegeneration in vivo. Proc Natl Acad Sci U S A 2014;111:9977-9982.

41. Chung CY, Seo H, Sonntag KC, Brooks A, Lin L, Isacson O. Cell type-specific gene expression of midbrain dopaminergic neurons reveals molecules involved in their vulnerability and protection. Hum Mol Genet 2005;14:1709-1725.
42. Gainetdinov RR, Fumagalli F, Wang YM, et al. Increased MPTP neurotoxicity in vesicular monoamine transporter 2 heterozygote knockout mice. J Neurochem 1998;70:1973-1978.

43. Taylor TN, Caudle WM, Shepherd KR, et al. Nonmotor symptoms of Parkinson's disease revealed in an animal model with reduced monoamine storage capacity. J Neurosci 2009;29:8103-8113.

44. Kilarski LL, Pearson JP, Newsway V, et al. Systematic review and UK-based study of PARK2 (parkin), PINK1, PARK7 (DJ-1) and LRRK2 in early-onset Parkinson's disease. Mov Disord 2012;27: 1522-1529.

45. Wilson GR, Sim JC, McLean C, et al. Mutations in RAB39B cause $\mathrm{X}$-linked intellectual disability and early-onset Parkinson disease with alpha-synuclein pathology. Am J Hum Genet 2014;95:729735 .

46. Inamdar AA, Hossain MM, Bernstein AI, Miller GW, Richardson JR, Bennett JW. Fungal-derived semiochemical 1-octen-3-ol disrupts dopamine packaging and causes neurodegeneration. Proc Natl Acad Sci U S A 2013;110:19561-19566.

47. Jiang H, Ren Y, Zhao J, Feng J. Parkin protects human dopaminergic neuroblastoma cells against dopamine-induced apoptosis. Hum Mol Genet 2004;13:1745-1754.

48. Tabrizi SJ, Orth M, Wilkinson JM, et al. Expression of mutant alpha-synuclein causes increased susceptibility to dopamine toxicity. Hum Mol Genet 2000;9:2683-2689.

49. Smith KM, Dahodwala N. Sex differences in Parkinson's disease and other movement disorders. Exp Neurol 2014;259:44-56.

50. Xiong N, Long X, Xiong J, et al. Mitochondrial complex I inhibitor rotenone-induced toxicity and its potential mechanisms in Parkinson's disease models. Crit Rev Toxicol 2012;42:613-632.

51. Zhang G, Xiong N, Zhang Z, et al. Effectiveness of traditional Chinese medicine as an adjunct therapy for Parkinson's disease: a systematic review and meta-analysis. PLOS ONE 2015;10: e0118498.

52. Fehr C, Sommerlad D, Sander T, et al. Association of VMAT2 gene polymorphisms with alcohol dependence. J Neural Transm (Vienna) 2013;120:1161-1169.

53. Penetar DM, Toto LH, Farmer SL, et al. The isoflavone puerarin reduces alcohol intake in heavy drinkers: a pilot study. Drug Alcohol Depend 2012;126:251-256.

54. Lin RC, Guthrie S, Xie CY, et al. Isoflavonoid compounds extracted from Pueraria lobata suppress alcohol preference in a pharmacogenetic rat model of alcoholism. Alcohol Clin Exp Res 1996;20: 659-663.

55. Eriksson AK, Lofving S, Callaghan RC, Allebeck P. Alcohol use disorders and risk of Parkinson's disease: findings from a Swedish national cohort study 1972-2008. BMC Neurol 2013;13:190.

56. Neiman J, Lang AE, Fornazzari L, Carlen PL. Movement disorders in alcoholism: a review. Neurology 1990;40:741-746.

57. Checkoway H, Powers K, Smith-Weller T, Franklin GM, Longstreth WT, Jr., Swanson PD. Parkinson's disease risks associated with cigarette smoking, alcohol consumption, and caffeine intake. Am J Epidemiol 2002;155:732-738. 